\title{
SEMI-GROUP GENERATED BY K-LIMITS
}

\author{
Bhant Quetho Frota Rezende, \\ Ricardo Apparicio Bacet \\ arlit \\ Rodney Carlos Bassanezi \\ IMECC. Lniversidade Estadual de Campinas, Brasil
}

\begin{abstract}
Wi une the defintions of limitoid, support and grill to present a se me-youp gereruted by the composition of some-k-limits character-

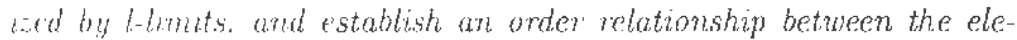
ments that ait obtatned.

KEYWORDS : Limitoid, stpport, grill, K-limits, semi-groups generated.
\end{abstract}




\section{Introduction}

The idea of st udying semi-groups generated by the operators of closure and interior initially appeared in the work of Kuratowski, in 1920 [12]. where the relationship among the fourteen elements are presented. Hammer later (in 1960. '111) presented an extension of Kuratowski's work. simplifying the initial hypotheses. Greco and Dolecki, following the ideas of Kuratowski. presinted in 1981 [5] a semi-group generated by isotonization and untitonization of the generalization of closure and interior operators. In 1975 De Giorgi and Frallzoni [4] developed the theory of $\Gamma$-convergence that has been successfully employed in approximation problems for Elliptic Partial Differential Equations, Variational Calculus, Minimax Problems, Convex Analysis and so forth. The definition of $\Gamma$-limit is closely related to the theory of lim inf and lim sup operators used by Francaviglia and Franzoni in $1983[6]$ in the study of the semi-group generated by

$$
\Gamma\left(X^{-}\right), \Gamma\left(X^{+}\right), \Gamma\left(Y^{+}, X^{-}\right) \text {and } \Gamma\left(X^{-}, Y^{+}\right) .
$$

In this work, we will present a semi-group generated by the composition of some $K$-limits characterized by $\Gamma$-limits, and we will establish an order rolationship between the elements that are obtained. We use the definition of the limituid. introduced by Greco in 1983 [7], and, in a more precise way. the definitions of limitoid support and grill that have been shown to be efficient tools for studying Kuratowski's $K$-limits.

\section{Preliminaries}

\section{$2.1 K$-operators and Systems of semifilter}

A topological space $X$ can be characterized by a system of neighborhoods or with the use of the interior operator. Let $\mathcal{N}(x)$ be the system of neighborhoods of $x, x \in X$. Then the following hold:

a) $\mathcal{N} \subset \mathcal{N}_{t}=\{A \subset X: x \in A\}$,

b) $\mathcal{N}^{2}=\mathcal{N}$.

c) $\mathcal{N}$ is a system of filters, i.e.:

$$
\mathcal{N} \neq \phi, \quad \phi \notin \mathcal{N}, \quad A \subset B \subset \bigcup_{A \in \mathcal{N}^{\prime}} A \Rightarrow B \in \mathcal{N} .
$$

On the other hand. if, for every set $A \subset X$ the interior of $A$ is indicated as $\operatorname{int}(\mathbf{A})$, then we have: 
is: $-4-2$ int $(4)$.

1): int ${ }^{2}(A)-\operatorname{int}(A)$

() $\operatorname{int}(A \cap B) \equiv \operatorname{int}(A) \cap \operatorname{int}(B)$. with the $\operatorname{int}(\phi)=\phi$ and $\operatorname{int}(X)=X$. 'T'lu' "orr" 'spondence between $N$ and int is given by:

$$
A \in N(x) \Leftrightarrow u \in \operatorname{int}(A)
$$

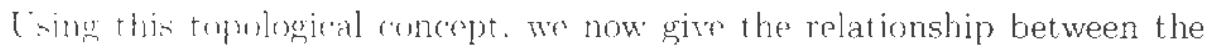

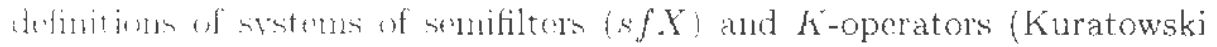
apdiatorist.

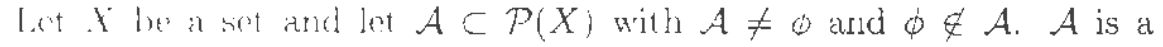
scuitilto' if

$$
A \in A, A \subset B \subset \bigcup_{A \in \mathcal{A}} \Rightarrow B \in \mathcal{A}
$$

$A$ sonifilter un $X$ is a somitilter $\mathcal{A}$ such that $X=\cup\{A: A \in \mathcal{A}\}$.

We itclicute by $x$. $X$ the set of the semifilters on $X$.

Let $X$ and $Y$ be two sets. A mapping $I: Y \rightarrow s f X$ is called systems uf semitilters on $X$ with domain $Y$. A set mapping given by:

$$
K: \mathcal{P}(X) \rightarrow \mathcal{P}(Y)
$$

jo denominated a $H$-operator from $X$ to $Y$. if

i) $h(0) \equiv \phi$ ind $h(X) \equiv Y$.

ii) $A \subset B \subset X \Rightarrow K(A) \subset K(B)$.

To any swstem of semifilters $\sigma$ there is associated the $K$-operator $K(\sigma)$ enfined by

$$
\sin (\sigma) \quad\{y \in Y: A \in \sigma(y)\} \text { for all } A \subset X \text {. }
$$

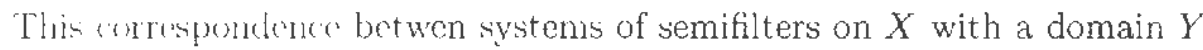
and $K$-operators from $X$ to $Y$ is one-to-one (injective), onto and it preserves 1he order as well. that in 10 say:

$$
A \in \Gamma(y) \Leftrightarrow y \in K(A)
$$

bexides.

$$
\Gamma<\mu \Leftrightarrow K(\Gamma) \subset K(\mu)
$$




\subsection{Grill}

If $\mathcal{A}$ and $\mathcal{B}$ are two families of sets, we will say that $\mathcal{A}$ is finer than $\mathcal{B}$ and we will write $\mathcal{A} \leq \mathcal{B}$ if for any $A \in \mathcal{A}$ there exists $B \in \mathcal{B}$ such that $B \subset A$. We will also say that $\mathcal{A}$ and $\mathcal{B}$ are equivalent and we will write $\mathcal{A} \equiv \mathcal{B}$ if $\mathcal{A} \leq \mathcal{B}$ and $\mathcal{B} \leq \mathcal{A}$.

Let $X$ be a set and let $\mathcal{A} \subset \mathcal{P}(X)$ be a non-degenerate family, that is, $\mathcal{A} \neq 0$ and $\phi \notin \mathcal{A}$. The grill of $\mathcal{A}$ is defined by

$$
\mathcal{A}^{\#}=\{A \subset X: A \cap F \neq \phi \text { for all } F \in \mathcal{A}\} .
$$

With respect to the grill operation, the following properties hold:

i) If $\mathcal{A} \subset \mathcal{P}(X)$ is a non-degenerate fanily, then $\mathcal{A}^{\# \#} \equiv \mathcal{A}$.

ii) If $\mathcal{A} \subset \mathcal{P}(X)$ and $\mathcal{B} \subseteq \mathcal{P}(Y)$ are semifilters of $X$ and $Y$ respectively, then $(\mathcal{A} \times \mathcal{B})^{\#} \supset \mathcal{A}^{\#} \times \mathcal{B}^{\#}$.

iii) If $\mathcal{A}$ and $\mathcal{B}$ are semifilters of $X$ such that $\mathcal{A} \subset \mathcal{B}$, then $\mathcal{B}^{\#} \subset \mathcal{A}^{\#}$.

iv) If $\mathcal{A} \subset \mathcal{P}(X)$ is a filter and $\mathcal{B} \subset \mathcal{P}(Y)$ is a semifilter such that

$$
\bigcap \mathcal{B} \equiv \equiv \text {, then }\left(\mathcal{A}^{\#} \times \mathcal{B}\right)^{\#} \subset \mathcal{A}^{\#} \times \mathcal{B}^{\#} \text {. }
$$

We can prove with respect to the grill operation, that

$$
h^{*}\left(\sigma^{\#}\right)(A)=\left(K(\sigma)\left(A^{c}\right)\right)^{c} .
$$

Therefore. if $\sigma$ is a system of neighborhoods of a topological space, then $K(\sigma)$ is the interior operator and $K\left(\sigma^{\#}\right)$ is the closure operator and we indicate $K(\sigma)$ by int $\sigma$ and $K\left(\sigma^{\#}\right)$ by $c l_{\sigma}$.

For the system of semifilters $\sigma: Y \rightarrow s f X$ the following statements are equivalent:

i) of is at svistom of filters;

b) $h(\sigma)(A \cap B)=K(\sigma)(A) \cap K^{\prime}(\tau)(B)$ for all $A, B \in \mathcal{P}(Y)$;

(c) $K^{-}\left(\sigma^{\#}\right)(A \cup B)=K^{-}\left(\sigma^{\#}\right)(A) \cup K^{\prime}\left(\tau^{\#}\right)(B)$ for all $A, B \in \mathcal{P}(Y)$.

\subsection{Limitoids and Lattices}

The prir $(L . \leq)$ made up by a set $L$ and an order relation " $\leq$ " is an ordered set. An ordered set is a complete lattice if each of its subsets pusisense's both an upper and a lower extreme.

Let $L_{1}$ and $L_{2}$ be complete lattice. A mapping $\Phi: L_{1} \rightarrow L_{2}$ is a complete hornomorphism if $\Phi(\sup W)=\sup (\Phi W)$ and $\Phi(\inf W)=\inf (\Phi W)$ for every subset $W \neq \phi$ of $L_{1}$. 
If $\therefore \quad L_{1}$ and $S$ rontains the inf $D$ and tho sup $D$ for every subset 1): ant s un ath that $S$ in a closed sublattion of $L_{1}$.

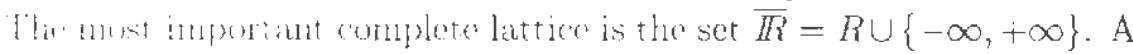

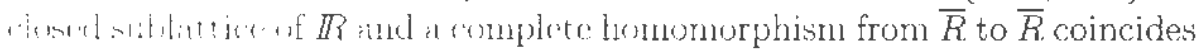

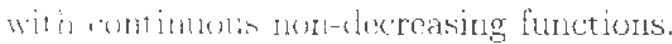

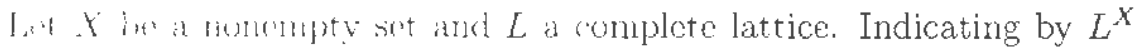

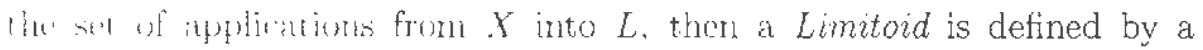
turtinal $T: L^{X} \rightarrow L$. such that for erery pair $(f, g) \in L^{X}$, and $\Phi$ a

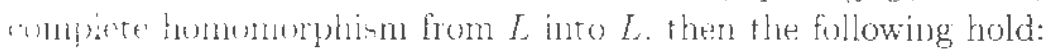

l. $f \leq g \Rightarrow T(f) \leq T(g)$ :

2) $T(\Phi \circ f)=\Phi(T(f))$ :

3) $T(f) \in \overline{f(X)}^{2}$, where $\overline{f(X)}^{l}$ is the smallest closed sublattice conailing $f(X)$.

Sunlut innurtant cximples of linnitoids can be exhibited by using finite (onntindtions of supremes and infina:

\section{Example:}

$$
T_{1}(f)=\inf _{y \in Y} \sup _{u \in X} f(x, y)=\inf _{Y} \sup _{X} f
$$

is: a limituist of $I . X \times Y$ inter $L$.

Analinguls:y.

$$
T_{2}(f)=\sup _{x \in X} \inf _{y \in Y} f(x, y)=\sup _{Y} \inf _{X} f^{\prime}
$$

in a limitoid.

In our work we center our interest on limitoids from $L^{X \times Y}$ into $L$ given by the [-]inits. as in the following:

$[\because] f: X \times Y-I R$ we defins

$$
\begin{aligned}
& \left(\Gamma\left(X^{\prime}\right) f\right)(x, y)=\limsup _{x \rightarrow x} f\left(x^{\prime}, y\right) \\
& \left(\Gamma\left(Y^{-}, f\right)(x, y)=\liminf _{y^{\prime}, y} f\left(x, y^{\prime}\right)\right. \\
& \left(\Gamma\left(Y^{-}, X^{+}\right) f\right)(x, y)=\inf _{A \in \mathcal{N}(x)} \liminf _{y^{\prime} \rightarrow y} \sup _{x^{\prime} \in A} f\left(x^{\prime}, y^{\prime}\right) \\
& \left(\Gamma\left(X^{+}, Y, f\right)(x, y)=\sup _{V \in \mathcal{N}(y) x^{\prime} \rightarrow x} \limsup _{y^{\prime} \in V} f\left(x^{\prime}, y^{\prime}\right)\right.
\end{aligned}
$$

lor $X$ and $Y$ topological spaces and for $\mathcal{N}(x)$ and $\mathcal{N}(y)$ as systems of neighborhoods on $X$ and $Y$ respectively, or, equivalently, as bases of filters. 
It is important to note that $\Gamma$-limits are fundamental for the study of minimax problems, due to the fact that the saddle-points can be defined by means of the topologies of $X$ and $Y$. We have the following results:

$$
\begin{aligned}
& \Gamma\left(Y^{-}\right) f \leq f \leq \Gamma\left(X^{+}\right) f \text { and } \\
& \Gamma\left(Y^{-}\right) f \leq \Gamma\left(X^{+}, Y^{-}\right) f \leq \Gamma\left(Y^{-}, X^{+}\right) f \leq \Gamma\left(X^{+}\right) f
\end{aligned}
$$

for all cxtended real function $f$. Besides, $f$ is lower semi-continuous on $Y$ if $f=\Gamma\left(Y, f\right.$. and, dually. $f$ is upper semi-continuous on $X$ if $f=\Gamma\left(X^{+}\right) f$. $\Gamma\left(Y^{*}\right) f$ and $\Gamma\left(X^{+} . Y^{-}\right) f$ are always lower semi-continuous on $Y$, while $\Gamma\left(X^{+}\right) f$ and $\Gamma\left(Y^{-}, X^{+}\right) f$ are upper semi-continuous on $X$.

In a general way, the $\Gamma$-limit of a function $f \in L^{X}$ is defined by a nlapping of the form:

$$
\Gamma\left(\mathcal{A}_{1}^{\alpha_{1}} \cdot \mathcal{A}_{2}^{\alpha_{2}} \ldots \mathcal{A}_{n}^{\alpha_{n}}\right): L^{X} \rightarrow L^{Y}
$$

with $\quad \Gamma\left(\mathcal{A}_{1}^{\gamma_{1}} \cdot \mathcal{A}_{2}^{\alpha_{2}} \ldots \mathcal{A}_{n}^{\alpha_{n}}\right) f=\operatorname{ext}_{\mathcal{A}_{n} \in \mathcal{A}_{n}}^{-\alpha_{n}} \ldots \operatorname{ext}_{A_{1} \in \mathcal{A}_{1}}^{-\alpha_{1}} \underset{x \in A_{1}}{\operatorname{ext}}{ }^{\alpha_{1}} \ldots \operatorname{ext}_{x \in A_{n}}^{\alpha_{n}}$

where $\mathcal{A}_{i}$ is a non-degenrate family of $X_{i}$, for each sub index $i,\left\{\alpha_{n}\right\}$ is a finite sequence of + or - signs and, moreover, ext ${ }^{-}=\inf$ and $\operatorname{ext}^{+}=$sup.

We then have that every $\Gamma$-limit is a limitoid [7].

The support of a limitoid. indicated by $S t(T)$, is a family of sets defined $b y$

$$
S t(T)=\left\{A \subset X: T\left(\chi_{A}\right)=1, \chi_{A} \in L^{X}\right\}
$$

where $\gamma_{A}$ is the characteristic step function of $A$.

We remark that the support of a limitoid is a semifilter [9].

Theorem 1: (The Representation of Limitoids)

If $L$ is a completely distributed complete lattice, then every limitoid is an inferior limit along a non-degenerate family. More precisely,

$$
T(f)=\lim _{s t(T)} \inf f[7] .
$$


Theorem 2: (Reticular Structure of Limitoids)

If $L$ is a completcly distributed conplete reticulate then the mapping

$$
\text { St }: \operatorname{Lim}(X, L) \rightarrow \operatorname{sf} X,
$$

which assuldaten 10 anch linitoid its support, is an isomorphism between the two reticulation 7 .

If thr limitrid is a [-limit, we then indicate the support of $\Gamma\left(\mathcal{A}_{1}^{\alpha_{1}}, \ldots, \mathcal{A}_{n}^{\alpha_{n}}\right)$ ly $\left(\mathcal{A}_{1}^{\prime i} \ldots \ldots A_{* \prime \prime}^{\prime \prime}\right)$. We will then have the following results:

a) $\left(\mathcal{A}^{-}\right) \leq \mathcal{A}$ and $\left(\mathcal{A}^{+}\right)=\mathcal{A}^{\#}$

b) $\left(\mathcal{A}_{1}^{\alpha_{1}} \ldots, \mathcal{A}_{n+1}^{\alpha_{n}-j}, \mathcal{A}_{n}^{-}\right)=\left(\mathcal{A}_{1}^{\alpha_{1}}, \ldots, \mathcal{A}_{n-1}^{\alpha_{n-1}}\right) \times \mathcal{A}_{n}$

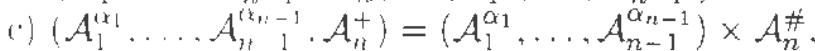

where. for any system of semifilters $\mathcal{A}$ and $\mathcal{B}$, we indicate by $\mathcal{A} \times \mathcal{B}$ the armitilen gonerated by the family $\{A \times B: A \in \mathcal{A}$ and $B \in \mathcal{B}$ ). Some particular cases cun be shown as follows:

$\left(\mathcal{A}, \mathcal{B}^{-}\right)=\mathcal{A} \times \mathcal{B}$.

$\left(\mathcal{A}^{+}, \mathcal{B}^{-}\right)=\mathcal{A}^{\#} \times B$

$\left(\mathcal{A}^{+}, \mathcal{B}^{+}\right)=(\mathcal{A} \times \mathcal{B})^{\#}$

$\left(\mathcal{A} \cdot \mathcal{B}^{+}\right)=\left(\mathcal{A}^{\#} \times \mathcal{B}\right)^{\#}$.

\section{Semi-Groups generated by $K$-Limits}

In $f: X \times Y \rightarrow R$, with $X$ and $Y$ as lopologleal spaces, with the topologies $\tau$ and $\sigma$. respectively. For $x \in X$ and $y \in Y$, lot $\mathcal{N}_{\tau}(x)$ and $\mathcal{N}_{\sigma}(y)$ bo the farnilies of neighborhooods of $x$ and $y$, respectively. For any pair $(n, 3)$ of signs + or - , the following $\Gamma$-limits

$$
\Gamma\left(\tau^{2} \cdot \sigma^{3}\right) f: X \times Y \rightarrow R
$$

can bxo defind by:

$$
\begin{aligned}
& \left(\Gamma\left(\tau^{\alpha}, \sigma^{\beta}\right) f\right)(x, y)=\Gamma\left(\mathcal{N}_{\tau}(x)^{\alpha}, \mathcal{N}_{\sigma}(y)^{\beta}\right) f \\
& \left(\Gamma\left(\tau^{\# \alpha}, \sigma^{\# \beta}\right) f\right)(x, y)=\Gamma\left(\mathcal{N}_{\tau}^{\#}(x)^{\alpha}, \mathcal{N}_{\sigma}^{\#}(y)^{\beta}\right) f
\end{aligned}
$$

Definition: K-linits

Let $A$ and $A^{\prime}$ be two subsets of the topolugical seets $X$ and $Y$. We then state that:

$$
\mu^{\prime}\left(\tau^{\alpha}, \sigma^{\prime}\right)(A)=A^{\prime} \Leftrightarrow \Gamma\left(\tau^{\alpha}, \sigma^{\beta}\right) \chi_{A}=\chi_{A^{\prime}}
$$


theresfore.

(i) $(x, y) \in K^{\circ}\left(\tau^{\alpha}, \sigma^{3}\right)(A) \Leftrightarrow A \in\left(\left(\mathcal{N}_{\tau}(x)^{\alpha}, \mathcal{N}_{\sigma}(y)^{\beta}\right)\right.$
(ii) $(x, y) \in K^{(}\left(\tau^{\# c}, \sigma^{\#,}\right)(A) \Leftrightarrow A \in\left(\left(\mathcal{N}_{\tau}^{\#}(x)^{\alpha}, \mathcal{N}_{\sigma}^{\#}(y)^{\beta}\right)\right.$

\section{Examples:}

1. $K\left(\tau^{-}, \sigma^{-}\right)(A)=$ int $A$ and $K\left(\tau^{+}, \sigma^{+}\right)=\operatorname{cl} A$

2. For $A \subset X \times Y$. and for any pairs of signs $(\alpha, \beta)$ and $(\bar{\alpha}, \bar{\beta})$. we have thit

$$
(x, y) \in\left(K\left(r^{\alpha}, \sigma^{j}\right) H\left(r^{\bar{\alpha}}, \sigma^{\bar{\beta}}\right)(A)\right) \Longleftrightarrow A \in \bigcup_{\Omega \in\left(\mathcal{N}_{r}(x)^{\bar{\alpha}}, \mathcal{N}_{\sigma}(y)^{\bar{\beta}}\right)} \bigcap_{(\bar{x}, \bar{y}) \in \Omega}
$$

$\left(\mathcal{N}_{r}(\bar{x})^{\alpha} \cdot \mathcal{N}_{\sigma}(\bar{y})^{\beta}\right)$

Concluding this part we may state that there exists a reticular isomorphism between the $K$-limits. the systems of semilfilters, and the $\Gamma$-limits, (on the sane set $X$ inj.

We will now establish the semi-group generated by the $K$-limits: $K\left(\tau^{-}, 0^{-}\right), K\left(\tau^{+}, 0^{-}\right), K\left(\tau^{-}, 0^{+}\right), K\left(\tau^{+}, 0^{+}\right), K\left(0^{+}, \tau^{-}\right), K\left(0^{-}, \tau^{-}\right)$, $K\left(\tau^{\#-}, 0^{\#-}\right)$ and $K\left(\tau^{\#+}, 0^{\#+}\right)$ where $\tau$ is topology in $X$ and 0 the chaotic topology in $Y$.

To obtain this semi-group we will need some results related to the de(omposition of the' $K$-limits.

To simplify our notation. we will use $K\left(\tau^{\alpha}\right)$ to represent $K\left(\tau^{\alpha}, i^{-}\right)$, where $i$ stands for the discreet topology in $X$.

Lemma 1: (Decomposition of the $K$-limits)

1) $K^{\prime}\left(\tau . \tau^{-}\right)=K^{-}\left(\tau^{-}\right) K^{-}\left(0^{-}\right)$

2) $K\left(\tau^{+} \cdot\left(0^{-}\right)=K^{*}\left(\tau^{+}\right) K^{*}\left(0^{-}\right)\right.$

3) $K\left(\tau^{-} \cdot\left(0^{+}\right)=K^{-}\left(\tau^{-}\right) K\left(0^{+}\right)\right.$

4) $K\left(\tau^{+},\left(0^{+}\right)=K^{+}\left(\tau^{+}\right) K\left(0^{+}\right)\right.$

5) $K\left(0^{-}, \tau^{-}\right)=K\left(0^{+}\right) K\left(\tau^{-}\right)$

6) $K\left(0^{-}, \tau^{+}\right)=K\left(0^{-}\right) K\left(\tau^{+}\right)$

7) $K\left(\tau^{\#-}, 0^{\#-}\right)=K\left(0^{+}\right) K\left(\tau^{+}\right)$

8) $K\left(\tau^{\#+} .0^{\#+}\right)=K\left(0^{-}\right) K\left(\tau^{-}\right)$

Lemma 2: For any sequence $\alpha_{1}, \alpha_{2}, \alpha_{3}$ and $\alpha_{4}$ of + or - signs, we have:

1) $K\left(0^{\alpha_{2}}\right) K\left(\sigma^{\alpha_{2}}\right) K\left(0^{\alpha_{3}}\right) K\left(\sigma^{\alpha_{4}}\right)=K\left(\sigma^{\alpha_{2}}\right) K\left(0^{\alpha_{3}}\right) K\left(\sigma^{\alpha_{4}}\right)$

2) $K\left(\sigma^{\alpha_{1}}\right) K\left(0^{\alpha_{2}}\right) K\left(\sigma^{\alpha_{3}}\right) K\left(0^{\alpha_{4}}\right)=K\left(\sigma^{\alpha_{1}}\right) K\left(\sigma^{\alpha_{3}}\right) K\left(0^{\alpha_{4}}\right)$ 
Lemma 3: For ar repronenting + or - signs. we hawe

$$
K\left(\sigma^{\alpha}\right) K^{\prime \prime}\left(\sigma^{\alpha}\right)=K^{\prime}\left(\sigma^{\alpha}\right) .
$$

Lemma 4: For ("wery pair $\alpha$ and $\beta$ as + or - signs, we have:

1) $\left.K\left(\sigma^{8}\right) h(0)^{3}\right) K\left(\sigma^{(8)}\right)=K\left(\sigma^{\beta}\right) K\left(\sigma^{\alpha}\right)$

2) $h\left(\sigma^{*}\right) h\left(\sigma^{i}\right) h\left(\sigma^{\prime 2}\right) h\left(\sigma^{\beta}\right)=h\left(\sigma^{\alpha}\right) h\left(\sigma^{\beta}\right)$

These lenmas are easily proven. We will, for example, ilustrate this witli the proof of $K\left(\tau^{-}\right) K\left(0^{+}\right) K\left(\tau^{-}\right)=K\left(0^{+}\right) K\left(\tau^{-}\right)$. To show this, we must prove that

$K\left(\tau^{-}\right) K\left(0^{+}\right) K\left(\tau^{-}\right) \Lambda=K\left(0^{+}\right) K\left(\tau^{-}\right) A$. that is.

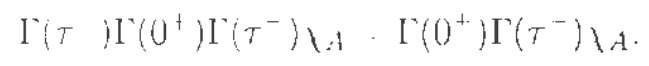

In fact, since we have:

$$
\begin{aligned}
& \Gamma\left(T^{-} \backslash A(l, y)=\sup _{l \in \mathcal{N}(x)} \inf _{x \in U} x_{\lambda}(\bar{x}, y)\right. \\
& \Gamma^{\prime}\left(0^{+}\right) \Gamma(\tau), f(x, y)=\sup _{y \in Y} \sup _{(i \in N(x)} \inf _{\bar{x} \in y^{\prime}} \chi A(\bar{x}, y)=h(x, y) \quad \text { and } \\
& \Gamma^{\prime}(\tau), \Gamma^{\prime}\left(0^{\prime}\right) \Gamma^{\prime}(\tau) h A(x, y)=\Gamma\left(\tau^{-}\right) h(x, y)=\operatorname{sip}_{U \subset N(x)} \inf _{\bar{x} \in U} h(\bar{x}, y)= \\
& =\sup _{U \in \mathcal{N}(x)} \inf _{\bar{x} \subset U} \sup _{y \subset Y} \sup _{\bar{U} \in \mathcal{N}(\bar{x})} \inf _{\overline{\bar{x}} \in \bar{U}} \chi_{A}(\overline{\bar{x}}, y)=j(x, y),
\end{aligned}
$$

then $j(x, y) \leq h(x, y)$

$\sin$

$$
\begin{aligned}
& f(x, y) \geq \sup _{l \in \mathcal{N}(x)} \inf _{\bar{x} \in \ell} \sup _{y \in Y} \inf _{\bar{x} \in l} \downarrow \rightarrow(\overline{\bar{x}}, y)
\end{aligned}
$$

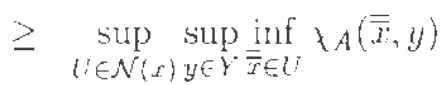

$$
\begin{aligned}
& \geq \operatorname{sip}_{y \in \gamma(\sin (x)} \inf _{\overline{\bar{x}} \in U} h(\overline{\bar{x}}, y)=h(x, y) \text {. }
\end{aligned}
$$

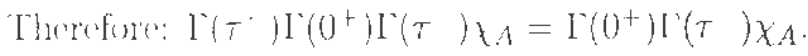

With the resilts of the previous lemmas, we can obtain the following: 
a) vight olements resulting from two compositions:

$$
\begin{array}{ll}
K\left(\tau^{+}\right) K\left(0^{-}\right) K\left(\tau^{-}\right) & K\left(\tau^{-}\right) K\left(\tau^{+}\right) K\left(0^{+}\right) \\
K\left(\tau^{+}\right) K\left(0^{+}\right) K\left(\tau^{-}\right) & K\left(\tau^{+}\right) K\left(\tau^{-}\right) K\left(0^{+}\right) \\
K\left(\tau^{-}\right) K\left(0^{-}\right) K\left(\tau^{+}\right) & K\left(\tau^{-}\right) K\left(\tau^{+}\right) K\left(0^{-}\right) \\
K\left(\tau^{-}\right) K\left(0^{+}\right) K\left(\tau^{+}\right) & K\left(\tau^{+}\right) K\left(\tau^{-}\right) K\left(0^{-}\right)
\end{array}
$$

b) cight elements resulting from three compositions:

$$
\begin{array}{ll}
K\left(\tau^{+}\right) K\left(\tau^{-}\right) K\left(0^{+}\right) K\left(\tau^{+}\right) & K\left(\tau^{+}\right) K\left(\tau^{-}\right) K\left(\tau^{+}\right) K\left(0^{+}\right) \\
K\left(\tau^{+}\right) K\left(\tau^{-}\right) K\left(0^{-}\right) K\left(\tau^{+}\right) & K\left(\tau^{+}\right) K\left(\tau^{-}\right) K\left(\tau^{+}\right) K\left(0^{-}\right) \\
K\left(\tau^{-}\right) K\left(\tau^{+}\right) K\left(0^{+}\right) K\left(\tau^{-}\right) & K\left(\tau^{-}\right) K\left(\tau^{+}\right) K\left(\tau^{-}\right) K\left(0^{+}\right) \\
K\left(\tau^{-}\right) K\left(\tau^{+}\right) K\left(0^{-}\right) K\left(\tau^{-}\right) & K\left(\tau^{-}\right) K\left(\tau^{+}\right) K\left(\tau^{-}\right) K\left(0^{-}\right)
\end{array}
$$

(c) four clements resulting from four compositions:

$$
\begin{aligned}
& K\left(\tau^{-}\right) K\left(\tau^{+}\right) K\left(\tau^{-}\right) K\left(0^{+}\right) K\left(\tau^{+}\right) \\
& K\left(\tau^{-}\right) K\left(\tau^{+}\right) K\left(\tau^{-}\right) K\left(0^{-}\right) K\left(\tau^{+}\right) \\
& K\left(\tau^{+}\right) K\left(\tau^{-}\right) K\left(\tau^{+}\right) K\left(0^{+}\right) K\left(\tau^{-}\right)
\end{aligned}
$$

which. added to the eight previously generated elements of the semi-group, make up the 28 elements.

Wo can also establish the following order relations in the semi-group:

a) among the generating elements of the semi-group:

$K(\tau, 0) \leq K\left(\tau^{\#+}, 0^{\#+}\right) \leq K\left(\tau^{+}, 0^{-}\right) \leq K\left(0^{-}, \tau^{+}\right) \leq K\left(\tau^{\#-}, 0^{\#-}\right) \leq$ $k^{-}\left(\tau^{+}, 0^{+}\right)$

and

$K^{\prime}\left(\tau^{\#+}, 0^{\#+}\right) \leq K\left(0^{+}, \tau^{-}\right) \leq K\left(\tau^{-}, 0^{-}\right) \leq K\left(\tau^{\#-}, 0^{\#-}\right)$.

These inequalities are easily obtained using grill properties.

b) among the elements resulting from two conıpositions:

$K^{\prime}\left(\tau^{+}\right) K^{-}(\tau) K^{-}\left(0^{-}\right) \leq K\left(\tau^{+}\right) K^{\prime}\left(0^{-}\right) K\left(\tau^{-}\right) \leq K\left(\tau^{+}\right) K\left(0^{+}\right) K\left(\tau^{-}\right) \leq$ $h^{*}\left(\tau^{+}\right) K^{*}(\tau) K^{*}\left(0^{+}\right)$

and

$K^{\prime}\left(\tau^{-}\right) K\left(\tau^{+}\right) K\left(0^{-}\right) \leq K\left(\tau^{-}\right) K\left(0^{-}\right) K\left(\tau^{+}\right) \leq K\left(\tau^{-}\right) K\left(0^{+}\right) K\left(\tau^{+}\right) \leq$ $K^{-}\left(\tau^{-}\right) K^{-}\left(\tau^{+}\right) K^{-}\left(0^{+}\right)$. 


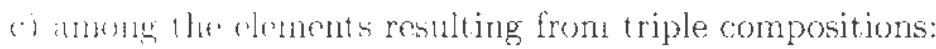

$$
\begin{aligned}
& \left.K(\tau) K^{\prime}\right) K(\tau) K(0) \leq K\left(\tau^{-}\right) K\left(\tau^{+}\right) K\left(0^{-}\right) K\left(\tau^{-}\right) \leq \\
& \leq K\left(\tau^{-}\right) K\left(\tau^{3}\right) K\left(0^{+}\right) K\left(\tau^{-}\right) \leq K\left(\tau^{-}\right) K\left(\tau^{+}\right) K\left(\tau^{-}\right) K\left(0^{+}\right)
\end{aligned}
$$

atid

$$
\begin{aligned}
& \left.K^{+}\right) K(\tau) h\left(\tau^{+}\right) K^{+}\left(0^{-}\right)<K^{+}\left(\tau^{+}\right) K^{+}\left(\tau^{-}\right) K^{*}\left(0^{-}\right) K^{+}\left(\tau^{+}\right) \leq \\
& \cdots h\left(\tau^{+}\right) K^{\prime}(\tau) K^{+}\left(0^{+}\right) K^{+}\left(\tau^{+}\right) \leq K^{\prime}\left(\tau^{+}\right) K^{\prime}\left(\tau^{-}\right) K\left(\tau^{+}\right) K\left(0^{+}\right)
\end{aligned}
$$

d) annong thuse alemernts resulting from four conpositions:

$$
\text { Kit }) K(\tau) K\left(\tau^{*}\right) K\left(0^{-}\right) K\left(\tau^{-}\right) \leq K\left(\tau^{+}\right) K\left(\tau^{-}\right) K\left(\tau^{+}\right) K\left(0^{+}\right) K\left(\tau^{-}\right)
$$

ind

$$
\left.M(\tau) K\left(\tau^{+}\right) K_{(\tau}\right) K\left(0^{-}\right) K\left(\tau^{+}\right) \leq K\left(\tau^{-}\right) K\left(\tau^{+}\right) K\left(\tau^{-}\right) K\left(0^{+}\right) K\left(\tau^{+}\right) .
$$

These relations are placed in the following table, where each line joining any 1 wo elements define an order relation between them, defiring the

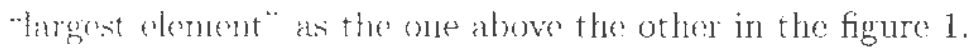

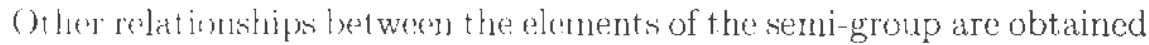
in wing the lemmont that follows:

Lemma 5: If $A$ and $B$ are elements of the generated scni-group and $A \subset B$, tho't):
a) $K(T) A \leq h\left(T^{+}\right) B$
1) $A \therefore B K\left(T^{+}\right)$
(अ) $A \leq K(T) B$
(1) $A K(T, B \leq B$
(1) $N\left(T^{-}\right) A \leq B$
f) $K\left(\tau^{\alpha}\right) A \leq K\left(T^{\alpha}\right) B$
g) $A K\left(\tau^{\alpha}\right) \leq B A\left(\tau^{i}\right)$, where o represents the + or - signs. 

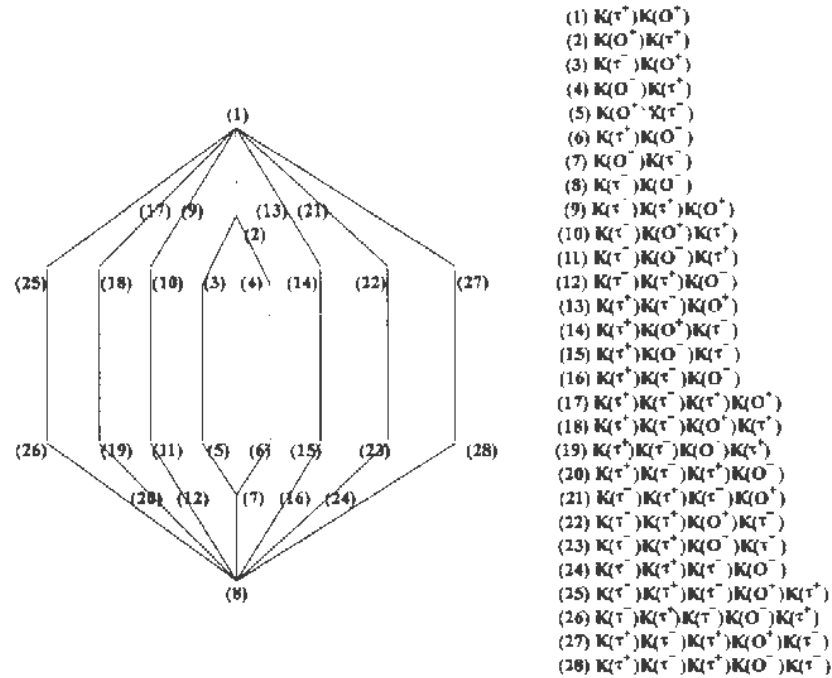

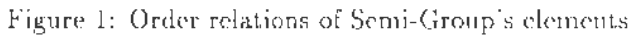

Finally we present a gennetric example of one of the ordor relations

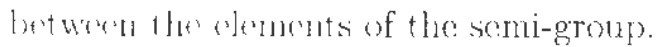

Wo have

$$
K\left(\tau^{+}\right) K\left(\tau^{-}\right) K\left(0^{-}\right) \leq K\left(\tau^{+}\right) K\left(0^{-}\right) K\left(\tau^{-}\right) .
$$

Comblering llat, $4 \subset X \times Y$. wo can see that

$$
(x, y) \in K\left(\tau^{+}\right) K\left(\tau^{-}\right) K\left(0^{-}\right) A \quad \text { if and only if }
$$

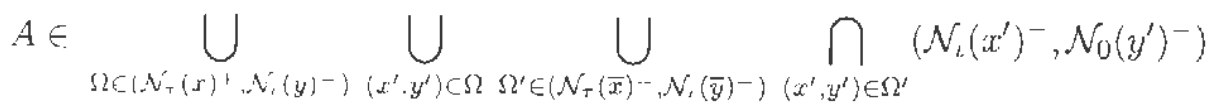


that is, if and only if

$$
4 \in \bigcup_{\Omega\left(A _ { r } \left(x^{\prime \prime} \times V_{i}(y)\right.\right.} \bigcap_{(\bar{x}, \bar{y}) \in \Omega\left(\Omega^{\prime} \in \mathcal{N}_{+}(\bar{r}) \times N_{1}(\bar{y})\right.} \bigcap_{\left(x^{\prime}, y^{\prime}\right) \in \Omega^{\prime}} \mathcal{N}_{\ell}\left(x^{\prime}\right) \times \mathcal{N}_{0}\left(y^{\prime}\right) .
$$

which means, in turn, that

A contains $\left\{\left(x^{\prime}, \mathbb{R}\right) \in \mathcal{N}_{t}\left(x^{\prime}\right) \times \mathcal{N}_{0}\left(y^{\prime}\right), \quad \forall\left(x^{\prime}, y^{\prime}\right) \in \Omega^{\prime}\right\}$ for some $\Omega^{\prime}, \Omega^{\prime}$ (wnt ains $\left\{B(\bar{x}, r) \times \bar{y} \in \mathcal{N}_{\tau}(\bar{x}) \times \mathcal{N}_{L}(\bar{y}), \quad \forall(\bar{x}, \bar{y}) \in \Omega,(\bar{y}=y)\right\}$ for some $\Omega$, and $\Omega$ contaius $\left\{(x, y) \in \mathcal{N}_{T}(x)^{H} \times \mathcal{N}_{t}(y)\right.$, with $\left.x_{n} \rightarrow x\right\}$.

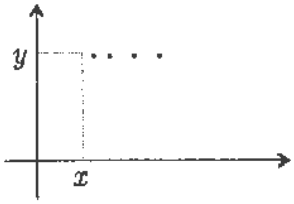

$\left(x_{m, y}\right) \rightarrow(x, y)$

figure 2.a

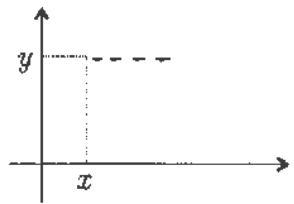

$B(\bar{x}, r) \times \bar{y}$

figure 2.b

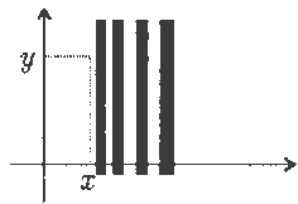

$\left(x^{\prime}, \mathbb{R i}\right)$

figure 2.c

Geometribally we can we that $A$ contains strips, as those in figure 2.c. We olsertor. as well, that

$$
(x . y) \in K^{*}\left(\tau^{+}\right) K\left(0^{-}\right) K\left(\tau^{-}\right) A \quad \text { if and only if }
$$

$$
d \in \bigcup_{\left\{\in \left[\mathcal{N}_{\tau}\left(x^{\prime}\right) \mathcal{N},(y ;)\right.\right.} \bigcap_{(\bar{x}, \bar{y}) \in \Omega} \bigcup_{\Omega^{\prime} \in\left(\mathcal{N}_{i}(\bar{x}), \mathcal{N}_{0}(\bar{y})-\right)} \bigcap_{\left\langle x^{\prime}, y^{\prime}\right) \in \Omega^{\prime}}\left(\mathcal{N}_{T}\left(x^{\prime}\right), \mathcal{N}_{l}\left(y^{\prime}\right)^{-}\right)
$$

which means that

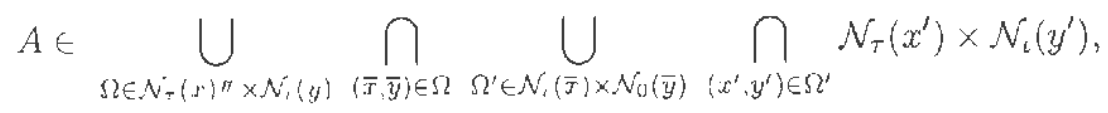

Whichumath that $A$ contaniss $\left\{B\left(x^{\prime}, \cdots\left(y^{\prime}\right)\right) \times y^{\prime} \in \mathcal{N}_{T}\left(x^{\prime}\right) \times \mathcal{N}_{L}\left(y^{\prime}\right), \forall\left(x^{\prime}, y^{\prime}\right) \in\right.$

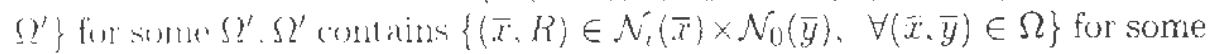
12. ancl $\left\{\right.$ contairs $\left\{\left(x_{n}, y\right) \subset \mathcal{N}_{7}(x)^{\#} \times \mathcal{N}(y)\right.$ with $\left.x_{n} \rightarrow x\right\}$. 


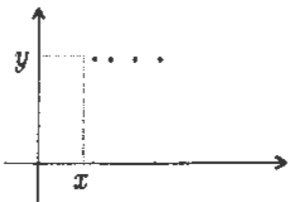

$\left(x_{n}, y\right) \rightarrow(x, y)$

figure 3.á

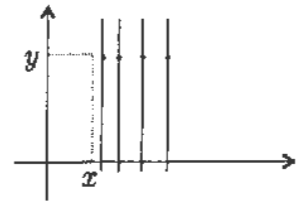

$(\bar{x}, \mathbb{R})$ figure 3.b

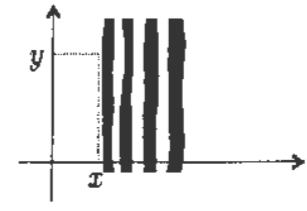

$B\left(x^{r}, r\left(y^{r}\right)\right) \times y^{r}$

figure 3.c

Again. we can, form the geometrical point of view, see that $A$ contains strips as those ir figure $3, c$; this means that if $(x, y) \in K\left(\tau^{+}\right) K\left(\tau^{-}\right) K\left(0^{-}\right) A$ then $(x, y) \in K\left(\tau^{+}\right) K\left(0^{-}\right) K\left(\tau^{-}\right) A$, that is, $K\left(\tau^{+}\right) K\left(\tau^{-}\right) K\left(0^{-}\right)$ $\leq K\left(\tau^{+}\right) K^{-}\left(0^{-}\right) K(\tau)$. 


\section{References}

I Ci. Binkholf. Lattice theory. An. Nath. Soc Coll. Publ., Provi-

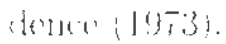

[2. Ci. Buttawo, Su una definizione generale dei $\Gamma$-limit, Boll. Un. Irat. Ital.. 5). 14-13. pp. 722-744. (1977).

35 Do Ginrot. I-colwergenza e G-convergenza, Boll. Un. Mat.

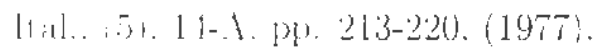

1. 1) Giurgi. T. Franzuni. Su un lipo di comgergenza variazionale, Atu. Ace. Vitz lineei Cl. Scj Fis Mat. Natur. (8) 58,6, pp. $842-850,(1975)$.

5. S. Dolecki. G. H. Groco. Cyrtologies of convergences, I, Relatório Trenico, Lib. Univ. Studi di Trento, (1983).

(j'Tl. Frasanni, Francaviglia. Suj I'-linait di due variabili. Relatório

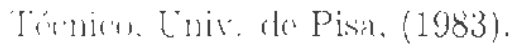

†. C. H. Cimen. Linnitoidi e roticoli completi. Ann. Univ. Ferrara, 29. pu. $153-164,(1983)$.

8) G. H. Groo. Linites, reticulados completamente distributivos e aplicacus as (onvergencias variacionais, IMECC, UNICAMP, (mini (14191). (1983).

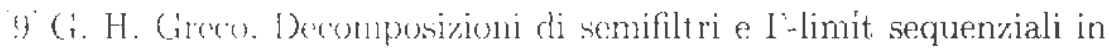
ret iroli contuletarnente distributivi; Ann. Mat. Pura a Appl., 4, 137. pp. 61-82, (1984).

[10] G. H. Groco, Teoria dos semifiltros, limites, reticulados completannon distributivos o convergências variacionais, 220 Seminário Brasiloiro do Análisos. (nini curso), 110 pgs. (1985)

:11 P. C. Hammor. Kuratowikis Closure Theorem, Nienw Archief Vou Wiskmude. 3. \%. pp. 74-80. (1960).

$[12$ K. Kuratowski. Su loperation $A$ de l'Analysis Situs. Fundarnenta Matturematicar. 3 (1922). 
Recoived: September 26. 1996.

\section{Eliane Quelho Frota Rezende}

Ricardo Apparicio Bacci

and

Rodney Carlos Bassanezi

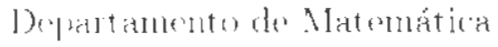

Institute de Matemática. Fistat listica e Computacao Cicntifica

Touversidial Estaclual do Campinas

Cidato Liniorstánia "Zeferino Vax"-Barao Geraldo

131081-970 Campinas-Sao Paulo

Brasil 